\title{
A retrospective study on risk factors and disease burden for hospital-acquired pneumonia caused by multi-drug-resistant bacteria in patients with intracranial cerebral hemorrhage
}

\author{
Haojun Yang ${ }^{1} \cdot$ Yishu Fan ${ }^{1} \cdot$ Chunhui $\mathrm{Li}^{2} \cdot$ Mengqi Zhang ${ }^{1} \cdot$ Weiping Liu ${ }^{1}$ (I)
}

Received: 29 May 2021 / Accepted: 1 November 2021 / Published online: 8 November 2021

(c) Fondazione Società Italiana di Neurologia 2021

\begin{abstract}
Purpose Hospital-acquired pneumonia (HAP) is becoming a serious problem in China, especially caused by multi-drug resistant (MDR), which is a risk factor for poor prognosis of intracranial cerebral hemorrhage (ICH). We investigate the risk factors for HAP among patients with ICH and study the antibiotic use and medical costs of MDR infection.

Methods We performed a retrospective, case-control, parallel study in Xiangya Hospital. Patients included in this study and diagnosed with basal ganglia hemorrhage were admitted between January 2017 and December 2019.

Results Univariate analysis discovered some personal risk factors including gender $(p=.002)$, age $(p=.023)$, and underlying conditions such as diabetes $(p=.036)$, coronary heart disease $(p=.009)$, and renal insufficiency $(p=.001)$. Invasive medical operations including endotracheal intubation, tracheotomy, ventilator use, lumbar puncture, urinary catheter insertion, and peripherally inserted central catheter (PICC) $(p<.001$ all) were also risk factors for HAP. Binary logistics regression indicated hospital duration, antibiotic exposure, and urinary catheter insertion explained $91.4 \%$ of the variance on HAP $(p<0.01)$. As for the antibiotic treatment, there were no difference in the duration of use days and total dose per patient between MDR and non-MDR group, except for Tigecycline. Antibiotic costs for the MDR group were significantly higher than those for the non-MDR group and no infection group $(p<0.001)$.

Conclusion To better prevent HAP particularly caused by MDR bacteria, we emphasize the aseptic technique especially in the management of equipment in patient care.
\end{abstract}

Keywords Intracranial cerebral hemorrhage $\cdot$ Hospital-acquired pneumonia $\cdot$ Multi-drug-resistant bacteria $\cdot$ Risk factors . Disease burden

\section{Introduction}

Hospital-acquired pneumonia (HAP) is not only one of the common complications but also a potential risk factor for poor prognosis of intracranial cerebral hemorrhage

Haojun Yang, Yishu Fan, Mengqi Zhang and Weiping Liu have contributed equally to this work.

Mengqi Zhang

zhangmengqi8912@163.com

Weiping Liu

Liuwp@csu.edu.cn

1 Department of Neurology, Xiangya Hospital of Central South University, Changsha 410008, Hunan, China

2 Infection Control Center, Xiangya Hospital, Central South University, Changsha Hunan 410008, China in intensive care unit (ICU), which consumes vast medical resources [1]. However, the incidence of HAP depends on the population studied. Previous studies have shown the incidence of HAP among patients with stroke, especially those characterized by advanced age, immune suppression, and long-term bed rest, can increase to $40 \%$ approximately $[2,3]$. The mortality rate of intracranial cerebral hemorrhage (ICH), as the most severe subtype of stroke, is around $29 \%$ within 1 year of onset $[4,5]$. The occurrence of HAP not only increases the expense of $\mathrm{ICH}$, but also aggravates the disease, which brings heavy financial and psychological burdens on patients' families [6]. Therefore, it is important to have a better understanding of the risk factors and disease burden of HAP in ICH patients, which is helpful to more timely and effective intervention so as to improve patients' survival and quality of life comprehensively. 
Due to the fact that HAP is the main cause of infection associated mortality in quite a few countries including China, increased use of antibiotic is common to see in hospitals [7]. In China, HAP is often treated with broadspectrum antibiotics empirically [8]. However, a increasing isolation rate of multi-drug resistant (MDR) organisms (MDROs) is noticed in most respiratory secretions from patients in ICU. In recent years, growing evidence has shown that MDROs has serious epidemic characteristics in different degrees in different provinces of China $[9,10]$. As a result, limited antibiotics can be used to treat HAP caused by MDROs, which means heavier social burdens than the burdens brought by HAP caused by non-drugresistant organisms.

Epidemiologic study of HAP among ICH patients is needed for infection prevention. Therefore, the aim of this study was to assess risk factors and disease burden resulting from HAP caused by MDRO infection among hospitalized patients treated for ICH.

\section{Materials and methods}

\section{Study design, population, inclusion, and exclusion criteria}

The retrospective study was conducted on patients with cerebral hemorrhage presenting with subsequent HAP from January 2017 to December 2019. All participants were adult patients admitted to Xiangya Hospital, a tertiary university hospital with approximately 100,000 admissions annually in China. Participants included were patients diagnosed with basal ganglia hemorrhage after first admission. Patients with multiple cerebral hemorrhages including basal ganglia hemorrhage at least were also included. Patients who were readmitted with cerebral hemorrhage were excluded. The included patients had no pulmonary infection on admission and had pulmonary infection on discharge. Patients diagnosed with HAP were defined as parenchymal lung infection that occurs $\geq 48 \mathrm{~h}$ after admission according to the definition in 2010, including ventilator associated pneumonia (VAP). Patients were divided into ICH-HAP group and ICH-non-HAP group. Additionally, ICH-HAP group were divided into multi-drug resistant (MDR) bacteria group and non-resistant bacteria group in terms of respiratory secretion detection. Data were extracted from the participants' medical records by using structured report formats. Demographic data, underlying conditions, treatments and procedures during admission, total dose, and duration of anti-HAP therapy were included in variables analysis. Each patient was assigned with a unique ID after abstracting record information from the patients' admission number.

\section{Detection of multi-drug-resistant bacteria}

The distiguishment of MDR bacteria were based on the results of respiratory secretion culture and drug sensitivity test. The "Technical Guidelines for the Prevention and Control of Multidrug-resistant Bacteria Nosocomial Infections (Trial)" promulgated by the Ministry of Health in 2011 clearly pointed out that MDR bacteria refer to bacteria that are resistant to 3 or more types of antimicrobial drugs used in clinical practice. The sputum specimens were inoculated on the nutrient medium under certain conditions such as temperature and humidity, in order to reproduce the bacteria with high nutritional requirements, including methicillin-resistant Staphylococcus aureus (MRSA), Vancomycin resistant enterococci (VRE), Acinetobacter baumannii (Ab), Pseudomonas aeruginosa, and Stenotrophomonas maltophilia. After drug susceptibility identification, MRSA is resistant to oxacillin or/and cefoxitin; VRE is resistant to Vancomycin; $\mathrm{Ab}$ and Pseudomonas aeruginosa are not sensitive to all antibacterial drugs; Stenotrophomonas maltophilia is resistant to cephalosporins, carbapenems, monocyclic $\beta$-lactams, and aminoglycoside antibiotics.

\section{Statistical analysis}

All demographic data were analyzed descriptively. Nominal data was presented as frequencies as well as percentages, and continuous data was presented as means and standard variations (mean $\pm \mathrm{SD}$ ). The differences between the mean scores in demographic characteristics were tested with two independent sample $t$-test. The chi-square test was used for comparison of different groups regarding the categorical variables. Regression analysis of binary logistics was used to elevate the risk of HAP among patients with cerebral hemorrhage. A $p$ value of less than 0.05 was considered statistically significant. Statistical analysis was performed using the SPSS 22.0 package program.

\section{Results}

\section{Risk factors for cerebral hemorrhage-HAP}

Univariate analyses were conducted to compare the differences between the cerebral hemorrhage-HAP group and cerebral hemorrhage-non-HAP group on demographic and clinical characteristics of the patients (Table 1). Patients with cerebral hemorrhage-HAP (mean age 58.11, range 23-90 years) were older than cerebral hemorrhage-non-HAP patients (mean age 54.78, range $15-86$ years) $(p=0.002)$. About $50 \%$ of the patients in the 
Table 1 Comparison of ICH-HAP and ICH-non-HAP groups in terms of the demographic and risk factors

\begin{tabular}{|c|c|c|c|c|c|c|}
\hline & \multicolumn{2}{|l|}{ ICH-HAP } & \multicolumn{2}{|l|}{ ICH-non-HAP } & \multirow[t]{2}{*}{ OR $(95 \% \mathrm{CI})$} & \multirow[t]{2}{*}{$P$ value } \\
\hline & $n(122)$ & $\%$ & $n(202)$ & $\%$ & & \\
\hline Male** & 94 & 77.05 & 121 & 59.90 & $2.247(1.353-3.732)$ & 0.002 \\
\hline Age $($ mean $\pm \mathrm{SD}) *$ & $58.11 \pm 12.95$ & & $54.78 \pm 12.55$ & & $(0.468-6.198)$ & 0.023 \\
\hline \multicolumn{7}{|l|}{ Age group } \\
\hline $18-40$ & 10 & 8.20 & 24 & 11.88 & $0.662(0.305-1.437)$ & 0.294 \\
\hline $41-64 * * *$ & 36 & 29.51 & 136 & 67.33 & $0.203(0.125-0.331)$ & $<0.001$ \\
\hline$>65 * * *$ & 76 & 62.30 & 42 & 20.79 & $6.294(3.819-10.732)$ & $<0.001$ \\
\hline Hospital duration (days) (mean $\pm \mathrm{SD}) * * *$ & $17.08 \pm 12.83$ & & $12.38 \pm 5.27$ & & $(2.247-7.072)$ & $<0.001$ \\
\hline \multicolumn{7}{|l|}{ Underlying conditions } \\
\hline Hypertension & 105 & 86.07 & 172 & 85.15 & $1.077(0.567-2.048)$ & 0.820 \\
\hline Diabetes mellitus* & 28 & 22.95 & 28 & 13.86 & $1.851(1.036-3.309)$ & 0.036 \\
\hline Hepatitis & 11 & 9.02 & 14 & 6.93 & $1.331(0.584-3.033)$ & 0.495 \\
\hline Coronary heart disease** & 23 & 18.85 & 18 & 8.91 & $2.375(1.233-4.661)$ & 0.009 \\
\hline Renal insufficiency*** & 18 & 14.75 & 9 & 4.46 & $3.712(1.610-8.554)$ & 0.001 \\
\hline \multicolumn{7}{|l|}{ During hospital admission } \\
\hline Used antibiotics* & 116 & 95.08 & 26 & 12.87 & $130.872(52.253-327.777)$ & 0.020 \\
\hline Used immunosuppressant & 0 & 0.00 & 0 & 0.00 & - & - \\
\hline Used glucocorticoids*** & 76 & 62.30 & 16 & 7.92 & $19.21(10.246-36.002)$ & $<0.001$ \\
\hline \multicolumn{7}{|l|}{ Invasive procedures during hospitalization } \\
\hline Tracheal intubation $* * *$ & 37 & 30.33 & 2 & 0.99 & $43.529(10.259-184.705)$ & $<0.001$ \\
\hline Incision of trachea*** & 35 & 28.69 & 1 & 0.50 & $80.862(10.904-599.659)$ & $<0.001$ \\
\hline Lumbar puncture*** & 50 & 40.98 & 21 & 10.40 & $5.985(3.357-10.671)$ & $<0.001$ \\
\hline Urinary catheter insertion*** & 102 & 83.61 & 51 & 25.25 & $15.100(8.498-26.832)$ & $<0.001$ \\
\hline $\mathrm{PICC}^{* * *}$ & 58 & 47.54 & 6 & 2.97 & $29.604(12.198-71.847)$ & $<0.001$ \\
\hline CVC & 6 & 4.92 & 2 & 0.99 & $5.172(1.027-26.047)$ & 0.066 \\
\hline Hematoma drainage tube $* * *$ & 3 & 2.46 & 51 & 25.25 & $0.075(0.023-0.245)$ & $<0.001$ \\
\hline Invasive ventilator assisted ventilation $* * *$ & 25 & 20.49 & 4 & 1.98 & $12.758(4.319-37.683)$ & $<0.001$ \\
\hline
\end{tabular}

${ }^{*} p$-value is $<0.05 ; * p$-value is $<0.01 ; * * *$-value is $<0.001$. (Mark of statistical significance is indicated in bold.)

hemorrhage-HAP group were over 58. The hemorrhageHAP group required longer periods of hospitalization (mean 17 versus 12 days) $(p<0.001)$, and suffered from more complications including diabetes mellitus $(22.95 \%$ versus $13.86 \%, \mathrm{OR}=1.851, p=0.036$ ), coronary heart disease $(18.85 \%$ versus $8.91 \%$, OR $=2.375, p=0.009)$, and renal insufficiency $(14.75 \%$ versus $4.46 \%$, OR $=3.712$, $p=0.001)$. Higher percentage of the patients in cerebral hemorrhage-HAP group received antibiotics $(95.08 \%$ versus $87.13 \%, \mathrm{OR}=2.856, p=0.02$ ) and glucocorticoids $(62.30 \%$ versus $7.92 \%, \mathrm{OR}=19.21, p<0.001)$ during the hospitalization compared with cerebral hemorrhage-nonHAP group.

The regression model established to explain the effects of variables on HAP has been demonstrated in Table 2. The results of binary logistics regression indicated that three predictors explained $91.4 \%$ of the variance on HAP. According to the results, the effect of hospital duration on HAP was statistically significant $(p=0.003)$, which explained $11.5 \%$ of the total variance. The effect of antibiotic exposure on HAP was statistically significant $(p<0.001)$, which explained $49.7 \%$ of the total variance. The effect of urinary
Table 2 Regression analysis of binary logistics showing the association of risk factors with cerebral hemorrhage-HAP

\begin{tabular}{lllllllll}
\hline Variable & B & S.E & Wals & df & Sig & Exp(B) & $\begin{array}{l}\text { Predicted } \\
\text { percentage } \\
\text { correct }\end{array}$ \\
\hline Hospital duration (days) & -0.115 & 0.039 & 8.530 & 1 & 0.003 & 0.891 & 91.4 \\
Antibiotic exposure & -0.497 & 0.645 & 59.437 & 1 & $<0.001$ & 0.007 & \\
Urinary catheter insertion & 0.143 & 0.033 & 19.211 & 1 & $<0.001$ & 1.154 & \\
\hline
\end{tabular}


catheter insertion on HAP was also statistically significant $(p<0.001)$, which explained $3.3 \%$ of the total variance.

\section{Antimicrobials use in HAP}

A total of $95.90 \%$ cerebral hemorrhage-HAP and $12.87 \%$ the cerebral hemorrhage-non-HAP patients received one or more antimicrobial therapy. Univariate analyses were conducted to compare the differences between patients of cerebral hemorrhage-HAP caused by drug-resistant bacteria and non-resistant bacteria on antimicrobial therapy (Table 3). The top three drug-resistant pathogens causing HAP among patients with cerebral hemorrhage were acinetobacter baumannii $(40.48 \%)$, klebsiella pneumoniae (26.19\%), and pseudomonas aeruginosa $(23.81 \%)$ according to results of respiratory secretion detection. The top four antimicrobials taken by cerebral hemorrhage-HAP patients caused by drug-resistant bacteria during hospitalization were Cefoperazone sulbactam (61.90\%), Piperacillin tazobactam (52.38\%), Meropenem (52.38\%), and Vancomycin (40.48\%). A total of $28.57 \%$ patients with cerebral hemorrhage-HAP caused by drug-resistant bacteria were treated with Tigecycline, and none patients with HAP caused by non-resistant bacteria were treated with Tigecycline. Except for Tigecycline, there were no statistically differences of duration of use days and total dose of per patient in other antimicrobials we collected.

\section{Expense of antibiotic treatment}

The mean expense of antibiotic treatment for patients with cerebral hemorrhage-HAP caused by MDR bacteria was $32,849.62 \pm 5068.80(\$ 5081.84 \pm 784.14)$, which was significantly higher than that of patients with
Table 3 Comparison of antimicrobial therapy between patients of cerebral hemorrhageHAP caused by multi-drug resistant bacteria and nonresistant bacteria

\begin{tabular}{|c|c|c|c|c|c|c|}
\hline \multirow[t]{2}{*}{ Characteristics } & \multicolumn{2}{|c|}{$\begin{array}{l}\text { HAP caused by } \\
\text { MDR bacteria } \\
(n=42)\end{array}$} & \multicolumn{2}{|c|}{$\begin{array}{l}\text { HAP caused by } \\
\text { non-MDR bacteria } \\
(n=80)\end{array}$} & \multirow[t]{2}{*}{ OR $(95 \% \mathrm{CI})$} & \multirow[t]{2}{*}{$P$ value } \\
\hline & $\begin{array}{l}\text { No./days/ } \\
\text { dose (g) }\end{array}$ & $\%$ & $\begin{array}{l}\text { No./days/ } \\
\text { dose(g) }\end{array}$ & $\%$ & & \\
\hline Cefoperazone sulbactam & 26 & 61.90 & 13 & 16.25 & $8.375(3.542-19.804)$ & $<0.001$ \\
\hline Piperacillin tazobactam & 22 & 52.38 & 39 & 48.75 & $1.156(0.548-2.442)$ & 0.703 \\
\hline Meropenem & 22 & 52.38 & 19 & 23.75 & $3.532(1.595-7.820)$ & 0.001 \\
\hline Vancomycin & 17 & 40.48 & 12 & 15.00 & $3.853(1.615-9.194)$ & 0.002 \\
\hline Tigecycline & 12 & 28.57 & 0 & 0.00 & & - \\
\hline Fluconazole & 10 & 23.81 & 4 & 5.00 & 5.938 (1.734-20.332) & 0.005 \\
\hline Ceftriaxone & 9 & 21.43 & 17 & 21.25 & $1.011(0.406-2.514)$ & 0.982 \\
\hline Levofloxacin & 8 & 19.05 & 6 & 7.50 & $2.902(0.934-9.017)$ & 0.057 \\
\hline Ceftazidime & 5 & 11.90 & 16 & 20.00 & $0.541(0.183-1.596)$ & 0.260 \\
\hline
\end{tabular}

Fig. 1 Comparison of costs of antibiotic treatment among patients with ICH-HAP caused by MDR bacteria (Group A) and non-MDR bacteria (Group B) and patients with cerebral hemorrhage-non-HAP (Group C)

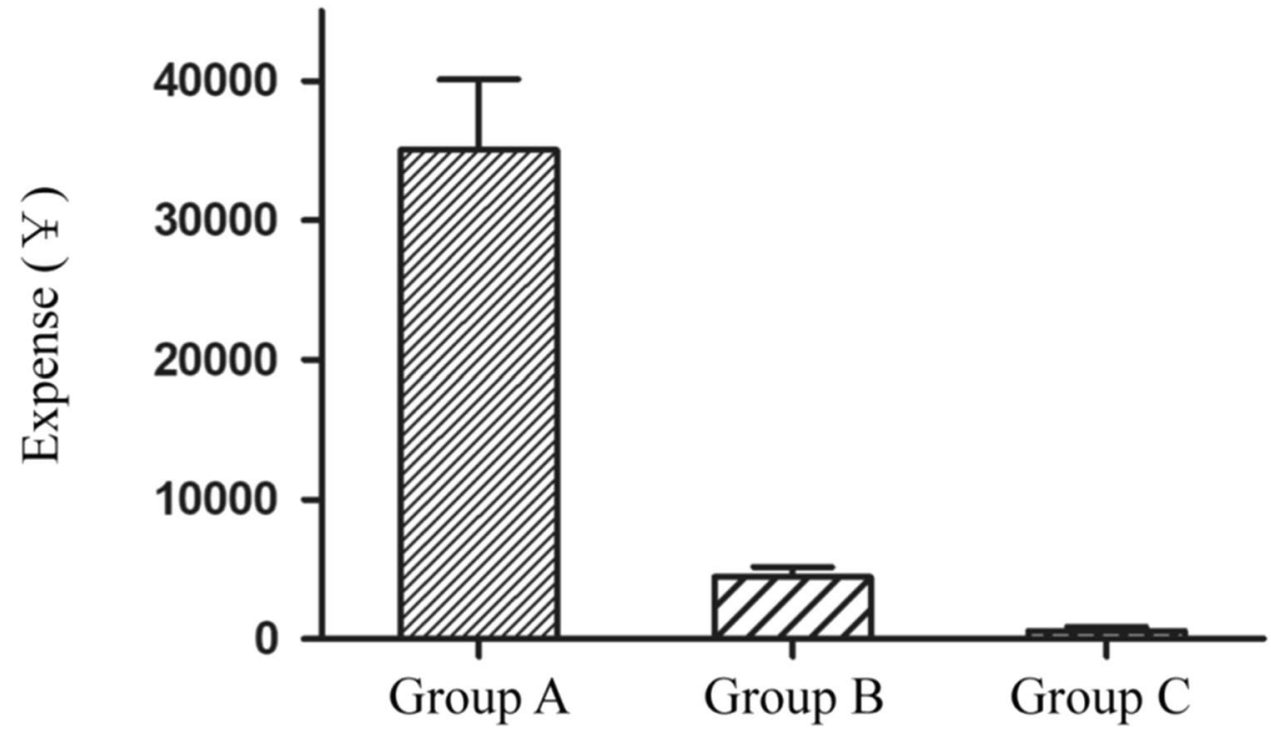


cerebral hemorrhage-HAP caused by non-resistant bacteria $(6242.05 \pm 697.88(\$ 965.65 \pm 107.96))$ and patients with cerebral hemorrhage-non-HAP $(567.34 \pm 298.67$ $(\$ 87.77 \pm 46.20))(p$ values were all $<0.001)($ Fig. 1$)$.

\section{Discussion}

HAP, one of the serious complications in patients with cerebral hemorrhage, is the major cause of multiple organ failure and death among patients with cerebral hemorrhage. Previous studies have shown the 30-day fatality rate of patients with acute stroke complicated with lower respiratory tract infection was almost three times higher than those uninfected [11]. Studies have shown that in ICH patients, pneumonia is associated with an increase in morbidity, length of stay, and mortality. The mortality rate of pneumonia patients (25.6\%) is higher than that of non-pneumonia patients (12\%) [12]. As a result, it is necessary to focus on the risk factors associated with HAP, so as to reduce the incidence of HAP and improve the prognosis. This study is one of the first to elevate risk factors and economic burden for hospital-acquired pneumonia caused by MDR bacteria among patients with ICH in Southern China.

The incidence of ICH has shown a clear upward trend in recent years and age performs as an independent risk factor for the occurrence of ICH. Risk factors of HAP among patients with ICH can be divided into personal factors and medical operation factors. The destruction of patient's selfdefense system increases the possibilities of suffering from HAP. In this study, univariate analysis of the two case-control groups discovered some personal factors including gender $(p=0.002)$, age $(p=0.023)$, and underlying conditions such as diabetes $(p=0.036)$, coronary heart disease $(p=0.009)$, and renal insufficiency $(p=0.001)$. Mean age of patients with ICH and HAP was 58.11 \pm 12.95 , which was significantly higher than the ICH-non-HAP group $(54.78 \pm 12.55)$, indicating age could be an important risk factor for ICH patients with HAP [13, 14]. Basal metabolism, physiological function, and self-regulation function decrease significantly with age. Growing evidence has shown diabetes is one of the risk factors for HAP in patients with ICH [15]. An elevated level of blood glucose, especially during the period of acute cerebral hemorrhage, combined with a long-term history of diabetes, leads to the patient's condition worse, which is more likely to induce HAP. Besides diabetes, coronary heart disease and renal insufficiency were also found to be associated with HAP in this study, which was scarcely reported [16-18].

Invasive operations are risk factors of HAP, including endotracheal intubation, tracheotomy, and ventilator use [19-21], which can also be found in our study.
These operations destroy the respiratory barrier. Airway mucosa becomes dryer without humidification and filtration of inhaled air in the upper airway, which obstructs the discharge of secretions and increases the risk of bacteria being inhaled and fixed into the lower respiratory tract [22]. Therefore, indications of tracheal intubation and incision should be controlled strictly so as to reduce the possibilities of iatrogenic infection. Furthermore, lumbar puncture $(p<0.001)$, urinary catheter insertion $(p<0.001)$, and peripherally inserted central catheter (PICC) $(p<0.001)$, which were common to see during the treatment of ICH, were also found to be risk factors associated with HAP statistically. Although these invasive procedures cannot cause HAP directly, they can suppress immune function by causing intracranial infection and urinary tract infection $[23,24]$, which emphasizes the importance of safe practice, especially the management of equipment in patient care, such as the aseptic technique in catheter use. Interestingly, hematoma drainage tube insertion was found to be a protective factor associated with $\operatorname{HAP}(p<0.001)$. This may be because timely drainage can reduce the local inflammatory response, thereby reducing the occurrence of adverse prognostic events. On the other hand, patients with hematoma drainage often have greater intracranial hemorrhage, worse systemic conditions, and lower immunity.

HAP not only prolongs hospitalization time, but also reduces the effect of rehabilitation, which is a both economic and psychological burden to patients and society. To increase the clinical cure rate and reduce the mortality rates caused by HAP, the importance of appropriate broadspectrum antibiotic treatment has been stressed during the management of HAP [25]. MDR bacteria, an important source of pathogens for iatrogenic infection, undoubtedly have a more adverse effect on the prognosis of patients with ICH. In this study, there is no difference in the duration of use days and total dose per patient between two groups of patients with ICH-HAP caused by MDR bacteria and non-drug resistant bacteria, except for Tigecycline. Tigecycline, the first generation of glycimecycline antibiotics with broad-spectrum activity, is suitable for patients with severe clinical infections, especially against MDR bacteria $[26,27]$. The method of short-duration, high-dose antibiotic courses seems useful to reduce the risk of antibiotic resistance [28].

However, there are some limitations in this study. We cannot assess whether there was a patient-to-patient infection transmission. If there were any epidemic outbreaks during the study period, the results would be affected undoubtedly. In addition, the small number of patients with ICH and HAP caused by MOD bacteria involved in this study is also a limitation. 


\section{Conclusion}

This study suggests that HAP may be associated with gender, age, hospital duration, diabetes, cardiovascular and urinary system diseases, and antibiotic and glucocorticoid exposure among patients with ICH. Further, some invasive operations including tracheal intubation, trachea incision, lumbar puncture, PICC, and invasive ventilator assisted ventilation are risk factors. Based on these results, to better prevent HAP particularly caused by MDR bacteria, we emphasize the aseptic technique especially in the management of equipment in patient care. Further, it is necessary to avoid excessive use of Tigecycline.

Author contribution This idea was come up with Dr. Zhang and Dr. Liu, and this manuscript was written by both authors.

Funding This research was funded by the Natural Science Foundations for Excellent Young Scholars of Hunan Province (No. 2021JJ20095), the Key Research and Development Program of Hunan Province (No. 2020SK2063), Research Project on Education and Teaching Innovation of Central South University (2021jy 145), the Natural Science Foundations of Hunan Province (Nos. 2020JJ4893 and 2020JJ4134), and the National Natural Science Foundation of China (No. 81501025).

Data availability Not applicable.

Code availability Not applicable.

\section{Declarations}

Ethical statement This study was approved by the ethics committee of Xiangya Hospital of Central South University. All data were confidential. Participants provided informed consent before participating in the study and were allowed to withdraw from the study at any phase.

Conflict of interest The authors declare no competing interests.

\section{References}

1. Leone M, Bouadma L, Bouhemad B, Brissaud O, Dauger S, Gibot $\mathrm{S}$ et al (2018) Hospital-acquired pneumonia in ICU. Anaesth Crit Care Pain Med 37(1):83-98

2. Lyons PG, Kollef MH (2018) Prevention of hospital-acquired pneumonia. Curr Opin Crit Care 24(5):370-378

3. Wałaszek M, Kosiarska A, Gniadek A, Kołpa M, Wolak Z, Dobroś W et al (2016) The risk factors for hospital-acquired pneumonia in the intensive care unit. Przegl Epidemiol 70(1):15-20-107-10

4. Masrur S, Smith EE, Saver JL, Reeves MJ, Bhatt DL, Zhao X et al (2013) Dysphagia screening and hospital-acquired pneumonia in patients with acute ischemic stroke: findings from get with the guidelines-stroke. J Stroke Cerebrovasc Dis 22(8):e301-e309

5. Kim SJ, Han KT, Kim SJ, Park EC (2016) Association between results of quality assessment of long-term care facilities and hospital-acquired pneumonia in individuals with stroke or neurodegenerative disease in Korea. J Am Geriatr Soc 64(12):e311-e313
6. Kemmling A, Lev MH, Payabvash S, Betensky RA, Qian J, Masrur S et al (2013) Hospital acquired pneumonia is linked to right hemispheric peri-insular stroke. PLoS One 8(8):e71141

7. Li CH, Duan JP, Liu SD, Meng XJ, Fu CC, Zeng C et al (2017) Assessing the risk and disease burden of clostridium difficile infection among patients with hospital-acquired pneumonia at a university hospital in Central China. Infection 45(5):621-628

8. Qiao M, Ying GG, Singer AC, Zhu YG (2018) Review of antibiotic resistance in china and its environment. Environ Int 110:160-172

9. Patil S, Chen H, Zhang X, Lian M, Ren PG, Wen F (2019) Antimicrobial resistance and resistance determinant insights into multi-drug resistant gram-negative bacteria isolates from paediatric patients in China. Infect Drug Resist 12:3625-3634

10. Lv XT, Lu XW, Shi XY, Zhou L (2017) Prevalence and risk factors of multi-drug resistant tuberculosis in Dalian. China J Int Med Res 45(6):1779-1786

11. Katazan IL, Cebul RD, Husak SH, Dawson NV, Baker DW (2003) The effect of pneumonia on mortality among patients hospitalized for acute stroke. Neurology 60(4):620-662

12. Alsumrain M, Melillo N, Debari VA, Kirmani J, Moussavi M, Doraiswamy V, Katapally R, Korya D, Adelman M, Miller R (2013) Predictors and outcomes of pneumonia in patients with spontaneous intracerebral hemorrhage. J Intensive Care Med 28(2):118-23

13. Burton LA, Price R, Barr KE, McAuley SM, Allen JB, Clinton $\mathrm{AM}$ et al (2016) Hospital-acquired pneumonia incidence and diagnosis in older patients. Age Ageing 45(1):171-174

14. Kanzigg LA, Hunt L (2016) Oral health and hospital-acquired pneumonia in elderly patients: a review of the literature. J Dent Hyg 90(Suppl 1):15-21

15. Wu HP, Chu CM, Lin CY, Yu CC, Hua CC, Yu TJ et al (2016) Liver cirrhosis and diabetes mellitus are risk factors for Staphylococcus aureus infection in patients with healthcare-associated or hospital-acquired pneumonia. Pulm Med 2016:4706150

16. Habayeb H, Sajin B, Patel K, Grundy C, Al-Dujaili A, Van de Velde S (2015) Amoxicillin plus temocillin as an alternative empiric therapy for the treatment of severe hospital-acquired pneumonia: results from a retrospective audit. Eur J Clin Microbiol Infect Dis 34(8):1693-1699

17. Zhu J, Zhang X, Shi G, Yi KH, Tan XR (2015) Atrial fibrillation is an independent risk factor for hospital-acquired pneumonia. PLoS One 10(7):e0131782

18. Wałaszek M, Kosiarska A, Gniadek A, Kołpa M, Wolak Z, Dobroś W et al (2016) The risk factors for hospital-acquired pneumonia in the intensive care unit. Przegl Epidemiol 70(1):15-20

19. Lioutas VA, Marchina S, Caplan LR, Selim M, Tarsia J, Catanese L et al (2018) Endotracheal intubation and in-hospital mortality after intracerebral hemorrhage. Cerebrovasc Dis 45(5-6):270-278

20. Gerakopoulos E, Walker L, Melling D, Scott S, Scott S (2019) Surgical management of multiple rib fractures reduces the hospital length of stay and the mortality rate in major trauma patients: a comparative study in a UK major trauma center. J Orthop Trauma 33(1):9-14

21. Luyt CE, Hékimian G, Koulenti D, Chastre J (2018) Microbial cause of ICU-acquired pneumonia: hospital-acquired pneumonia versus ventilator-associated pneumonia. Curr Opin Crit Care 24(5):332-338

22. Roquilly A, Marret E, Abraham E, Asehnoune K (2015) Pneumonia prevention to decrease mortality in intensive care unit: a systematic review and meta-analysis. Clin Infect Dis 60(1):64-75

23. Mu J, Ni C, Wu M, Fan WX, Liu Z, Xu FJ et al (2020) A retrospective study on risk factors for urinary tract infection in patients with intracranial cerebral hemorrhage. Biomed Res Int 2020:1396705 
24. Meng XJ, Liu SD, Duan JP, Huang X, Zhou PC, Xiong XR et al (2017) Risk factors and medical costs for healthcare-associated carbapenem-resistant Escherichia Coli infection among hospitalized patients in a Chinese teaching hospital. BMC Infect Dis 17(1):82

25. Freire AT, Melnyk V, Kim MJ, Datsenko O, Dzyublik O, Glumcher $\mathrm{F}$ et al (2010) Comparison of tigecycline with imipenem cilastatin for the treatment of hospital-acquired pneumonia. Diagn Microbiol Infect Dis 68(2):140-51

26. Cui N, Cai H, Li Z, Lu Y, Wang G, Lu A (2019) Tigecyclineinduced coagulopathy: a literature review. Int J Clin Pharm 41(6):1408-1413
27. Korth-Bradley JM, Troy SM, Matschke K, Muralidharan G, Fruncillo RJ, Speth JL et al (2012) Tigecycline pharmacokinetics in subjects with various degrees of renal function. J Clin Pharmacol 52(9):1379-1387

28. Stein RA (2008) When less is more: high-dose, short duration regimens and antibiotic resistance. Int J Clin Pract 62(9):1304-1305

Publisher's note Springer Nature remains neutral with regard to jurisdictional claims in published maps and institutional affiliations. 